\title{
Flavonols and Crown-Flavonols as Metal Cation Chelators. The Different Nature of $\mathrm{Ba}^{2+}$ and $\mathrm{Mg}^{2+}$ Complexes
}

\author{
A. D. Roshal,* A. V. Grigorovich, and A. O. Doroshenko \\ Institute of Chemistry of Kharkov State University, Kharkov 310077, Ukraine \\ V. G. Pivovarenko \\ Department of Chemistry, National Taras Shevchenko University, Kiev 252017, Ukraine
}

\author{
A. P. Demchenko \\ A. V. Palladin Institute of Biochemistry, Kiev 252030, Ukraine
}

Received: August 1, 1997; In Final Form: April 24, 1998

\begin{abstract}
The derivatives of 3-hydroxyflavone exhibit excited-state intramolecular proton transfer (ESIPT) reaction with significant $(60-80 \mathrm{~nm})$ shifts of fluorescence spectra between normal and phototautomer forms. This fact makes these compounds attractive as fluorescence probes in analytical chemistry, biophysics, and molecular biology. Different flavonol derivatives, including 4'-(monoaza-15-crown-5)flavonol, were synthesized, and their absorption and fluorescent spectra were studied in acetonitrile in the presence of different concentrations of $\mathrm{Mg}^{2+}$ and $\mathrm{Ba}^{2+}$ ions. It was shown that the general feature of flavonols is the ability to form two types of complexes with alkaline-earth cations: the low-stability "external" and high-stability chelating complexes. On the formation of the complexes, parent flavonols and their 4'-dialkylamino derivatives undergo different perturbations of their electronic structures. 4'-(Monoaza-15-crown-5)flavonol forms two types of complexes with both $\mathrm{Mg}^{2+}$ and $\mathrm{Ba}^{2+}$ ions; the sequence of steps in formation of $\mathrm{Ba}^{2+}$ and $\mathrm{Mg}^{2+}$ complexes is different.
\end{abstract}

\section{Introduction}

The derivatives of 3-hydroxyflavone recently have attracted the attention of many researchers because of their specific spectroscopic properties. They exhibit excited-state intramolecular proton transfer (ESIPT) reaction, ${ }^{1-3}$ which is accompanied by a very significant Stokes shift of about 9000 $\mathrm{cm}^{-1}$. The observation of two forms of emission, normal (NF) and proton transfer (PT), separated by $60-80 \mathrm{~nm}$ and of variation of relative intensities of these forms under the influence of different factors makes these compounds very attractive fluorescence probes for analytical chemistry, biophysics, and cellular biology. Thus, the ratio of intensities of these forms exhibits strong dependence on solvent polarity ${ }^{4}$ and hydrogen bonding with the solvent molecules. ${ }^{2,4,5}$ The sensitivity of fluorescence spectra of flavonols to the properties of their environment suggests their use as the probes for formation of micelles ${ }^{6,7}$ and as fluorochromic indicators for $\mathrm{Sc}^{3+}, \mathrm{Ga}^{3+}$, and $\mathrm{Th}^{3+}$ ions. $^{8}$

The most prospective in this respect are $4^{\prime}$-dialkylaminoflavonols. ${ }^{4,9}$ Their normal form of emission demonstrates significant charge-transfer character. Being very sensitive to perturbation by a dielectric environment, these and related aminoflavonols may serve the basis for creation of fluorescent indicators with high sensitivity and selectivity. The demand for compounds with such properties exists, in particular, in biochemical studies, where the nanomolar $\left(10^{-7} \mathrm{M}\right)$ concentrations of $\mathrm{Ca}^{2+}$ ions have to be determined in the presence of millimolar $\left(10^{-3} \mathrm{M}\right)$ concentrations of $\mathrm{Mg}^{2+}$ ions.

* To whom correspondence should be addressed. Institute of Chemistry of Kharkov State University, 4 Svobody Square, Kharkov 310077, Ukraine. Fax: 380-057-2-79-13-13 (tel: 380-057-2-45-73-35). E-mail: Alexandre.D.Rochal@univer.kharkov.ua.

\section{SCHEME 1}

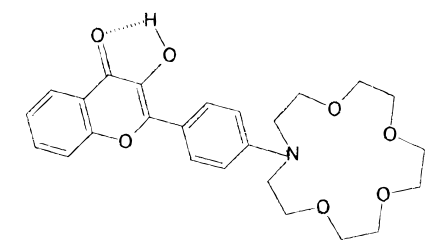

I

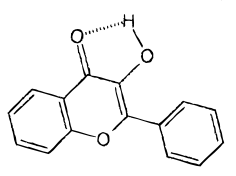

III
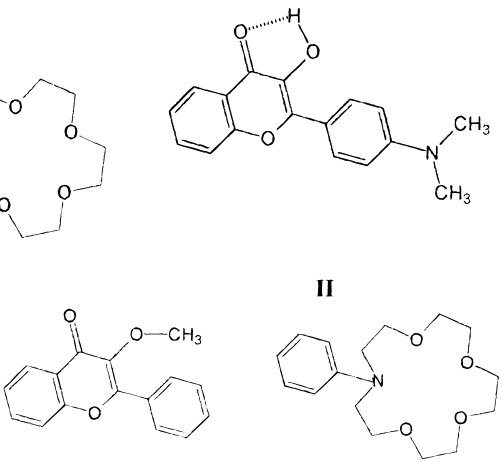

IV
II

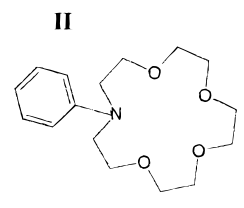

V
Recently we synthesized 4'-(15-azacrown-5)flavonol (I) ${ }^{10,11}$ (see structure in Scheme 1), which has the prospect of highly selective binding with cations of different charges, radii, and electronic properties and which changes dramatically its properties on complex formation. This molecule possesses two ion chelator sites, which are coupled with the system of conjugated double bonds of the chromophore system: ortho-groups, hydroxyl 3-OH and carbonyl $4-\mathrm{C}=\mathrm{O}$ form one site, and a crownmacrocycle at another side of molecule may serve as the second site. It is known that phenolic ortho-groups have a high affinity to multivalent cations of small radii, such as $\mathrm{Mg}^{2+}, \mathrm{Al}^{3+}$, or $\mathrm{Fe}^{3+}$, while the 15-member crown macrocycles have the highest affinity to alkaline and alkaline-earth metals of medium radius: $\mathrm{Na}^{+}, \mathrm{K}^{+}, \mathrm{Ca}^{2+}$, and $\mathrm{Sr}^{2+} .12$ Since one of the chelation sites is situated close to the negative, and the other to the positive end of molecular dipole, the complex formation is expected to result in different, probably opposite, effects on absorption and 
fluorescence spectra of this flavonol. An additional effect, the disappearance of the PT fluorescence band, should be observed on substitution by a metal cation of a proton belonging to the hydrophylic group 3-OH.

In the present study we investigated the absorption and fluorescence spectra of crown-flavonol $\mathbf{I}$ in acetonitrile in the presence of different concentrations of ions $\mathrm{Mg}^{2+}$ and $\mathrm{Ba}^{2+}$. These cations substantially differ by their ionic radius $r\left(r_{\text {cryst }^{-}}\right.$ $\left.\left(\mathrm{Ba}^{2+}\right) / r_{\text {cryst }}\left(\mathrm{Mg}^{2+}\right) \approx 1.9-2.2^{13}\right)$ and also by their ionization potential and electron affinity. The last property determines the difference in their electron-acceptor abilities and their abilities to polarize the $\pi$-electronic system of the chromophore. To understand in detail the processes that occur on complex formation of flavonol I, we studied also the interaction with the above-mentioned cations of other compounds which contain ion-binding centers of similar type: flavonols II-IV and phenyl-15-azacrown-5 (V). Since the spectra of flavonols are very sensitive to the presence of proton-donor impurities in the solvent, we studied also the influence of the addition of water on fluorescence spectra of flavonols $\mathbf{I}-\mathbf{I V}$ in acetonitrile. Our results demonstrate that ion binding to flavonol I proceeds in two steps, and the sequence of these steps is not the same in the cases of $\mathrm{Mg}^{2+}$ and $\mathrm{Ba}^{2+}$ ions.

\section{Experimental Section}

Flavonol Synthesis. The flavonols I-IV have been synthesized from 2-hydroxyacetophenone and the corresponding benzaldehydes by the Algar-Flynn-Oyamada reaction ${ }^{14,15}$ and purified by means of repeated recrystallization or column chromatography. All flavonols were homogeneous according to thin-layer chromatography (TLC) on Silufol UV-254 in chloroform-methanol $(95: 5,9: 1$, or $85: 15$, v/v). Their structures have been confirmed by quantitative elemental analysis, proton magnetic resonance (PMR), and UV-visible and infrared (IR) spectrometry (see the next subsection). Compound V, 98\% purity, was purchased from TCI American, Inc. (Portland, OR).

Spectroscopic Confirmation of Structure. PMR spectra were recorded on a Bruker WP-100 Fourier spectrometer at room temperature with tetramethylsilane as internal standard. The data presented below are on the $\delta$-scale and ordered as follows: signal position in ppm, form of signal, (coupling constant in $\mathrm{Hz}$ ), number of protons, and (their position in the molecule). Absorption spectra were recorded on a Hitachi U3210 instrument on $2 \times 10^{-5} \mathrm{M}$ solutions of flavonols in ethanol or in $0.01 \mathrm{M}$ Tris buffer, $\mathrm{pH}$ 7.4. Infrared (IR) spectra were recorded in a Pye Unicam SP3-300 instrument in pellets of $\mathrm{KBr}$. Data presented are in $\mathrm{cm}^{-1}$.

3-hydroxy-4'-N-(15-azacrown-5)flavone (I). PMR spectrum in $\mathrm{CDCl}_{3}$ : $8.98 \mathrm{~s}, 1 \mathrm{H}(\mathrm{OH}-3) ; 8.25 \mathrm{~m}, 1 \mathrm{H}(\mathrm{H}-5) ; 7.38 \mathrm{~m}, 1 \mathrm{H}(\mathrm{H}-$ $6) ; 7.63 \mathrm{~m}, 1 \mathrm{H}(\mathrm{H}-7) ; 7.55 \mathrm{~m}, 1 \mathrm{H}(\mathrm{H}-8) ; 8.17 \mathrm{~d}(J=9), 2 \mathrm{H}\left(\mathrm{H}-2^{\prime}, 6^{\prime}\right)$; $6.80 \mathrm{~d}(J=9), 2 \mathrm{H}\left(\mathrm{H}-3^{\prime}, 5^{\prime}\right) ; 3.68 \mathrm{~m}, 20 \mathrm{H}(\mathrm{CH} 2-\mathrm{CH} 2)$. IR spectrum: $1090,1120,1170-v(\mathrm{C}-\mathrm{O}) ; 1580-v(\mathrm{C}=\mathrm{O}) ; 1505-$ $v(\mathrm{C}=\mathrm{C}) ; 2860,2920-v\left(\mathrm{CH}_{2}\right)$. Absorption spectrum in ethanol: $\lambda_{\max }=408 \mathrm{~nm}\left(\epsilon=39900 \mathrm{dm}^{3} \mathrm{~mol}^{-1} \mathrm{~cm}^{-1}\right)$.

3-Hydroxy-4'-dimethylaminoflavone (II). PMR spectrum in DMSO: $9.16 \mathrm{~s}, 1 \mathrm{H}(\mathrm{OH}-3) ; 8.08 \mathrm{dd}\left(J_{1}=8 ; J_{2}=2\right), 1 \mathrm{H}(\mathrm{H}-5) ; 7.66-$ $7.77 \mathrm{~m}, 2 \mathrm{H}(\mathrm{H}-6,7) ; 7.43 \mathrm{~m}, 1 \mathrm{H}(\mathrm{H}-8) ; 8.13 \mathrm{~d}(J=9), 2 \mathrm{H}\left(\mathrm{H}^{\prime} 2^{\prime}, 6^{\prime}\right)$; $6.85 \mathrm{~d}(J=9), 2 \mathrm{H}\left(\mathrm{H}-3^{\prime}, 5^{\prime}\right) ; 3.00 \mathrm{c}, 6 \mathrm{H}\left(\mathrm{NCH}_{3}-4^{\prime}\right)$. Absorption spectrum in ethanol: $\lambda_{\max }=405 \mathrm{~nm}\left(\epsilon=39000 \mathrm{dm}^{3} \mathrm{~mol}^{-1}\right.$ $\left.\mathrm{cm}^{-1}\right)$.

Basic physical-chemical characteristics of flavonols II-IV correspond to those presented previously. ${ }^{14,16}$

Other Chemical Procedures. Acetonitrile, which was used as a solvent, was additionally purified, as described elsewhere, ${ }^{17}$ because the 3-hydroxyflavone derivatives are sensitive to the presence of water. In the studies of complex formation we used the anhydrous barium perchlorate from Aldrich and dehydrated magnesium perchlorate obtained by roasting of trihydrate sample at $215{ }^{\circ} \mathrm{C}$ and $0.15 \mathrm{mmHg}$ pressure during $3 \mathrm{~h} .{ }^{18}$ The quality of dehydration was controlled gravimetrically.

Spectroscopic Measurements. Absorption spectra were measured on a Hitachi U-3210 spectrophotometer. Fluorescence spectra were measured on a Hitachi F-4010 spectrofluorimeter at $20.0 \pm 0.1{ }^{\circ} \mathrm{C}$.

Ligand Concentrations, Methods of Titration. Affinity constants $K_{\mathrm{s}}$ and stoichiometric composition of the complexes were calculated from absorption spectra by iteration methods analogous to those described. ${ }^{19,20}$

Titrations were usually made by addition of small amounts of acetonitrile solution of investigated flavonol $\left(1 \times 10^{-5}\right.$ to 5 $\left.\times 10^{-5} \mathrm{~mol} / \mathrm{L}\right)$ and alkali-earth metal perchlorate $(2 \mathrm{~mol} / \mathrm{L})$ to the solution of the same flavonol in an initial concentration.

In the case of two complexes with similar $K_{\mathrm{s}}$ the dependency of extinction on metal concentration for one of the components was measured at isosbestic points produced by titration by the other component. ${ }^{21,22}$

Quantum-Chemical Calculations. Electronic structure of flavonols and their complexes with $\mathrm{Mg}^{2+}$ was calculated in full valent semiempirical approximation by the method PM3. ${ }^{23}$ The degree of redistribution of electronic density between different fragments of the studied molecules on transition from the ground to the excited state $(\Delta Q)$ was determined as the difference of total charges on atoms which compose these fragments in the $\mathrm{S}_{0}$ and $\mathrm{S}_{1}$ states:

$$
\Delta Q=\sum q_{i} \mathrm{~S}_{1}-\sum q_{i} \mathrm{~S}_{0}
$$

where $q_{i}$ are the charges on atoms that are included in the particular fragment.

\section{Results and Discussion}

The spectroscopic properties of flavonol derivatives depend significantly on intramolecular hydrogen bonding in their molecules. On transition to the excited state, the basicity of the carbonyl group and the acidity of the hydroxy group increase. This fact favors the proton-transfer reaction, so the fluorescence spectra of flavonols consist of two well-resolved emission bands. The ratio of intensities of these forms depends not only on experimental conditions but also on the structure of the flavonol molecule. Thus in the spectrum of flavonol III in acetonitrile the emission intensity of the phototautomer form is higher by $1-2$ orders of magnitude than the intensity of the normal form, while in the emission spectra of dialkylamino derivatives I and II the fluorescence intensities of the two forms are similar with even somewhat lower phototautomer emission. The latter result may be due to more significant increase of hydroxylic group 3-OH acidity in the excited state of dialkylamino derivatives as a result of the stabilizing influence of $\pi$-electron-donor substituents in the $4^{\prime}$ position.

Intermolecular Hydrogen Bonding with Water. In flavonol solutions in both proton-donor and proton-acceptor solvents the intramolecular hydrogen bonds may compete with intermolecular bonds with solvent molecules. This competition hampers the proton-transfer reaction and results in the decrease of phototautomer emission up to its complete disappearance (as it was observed for dimethylaminoflavonol in alcohols ${ }^{24}$ ). This fact and the possibility of significant influence of trace amounts 


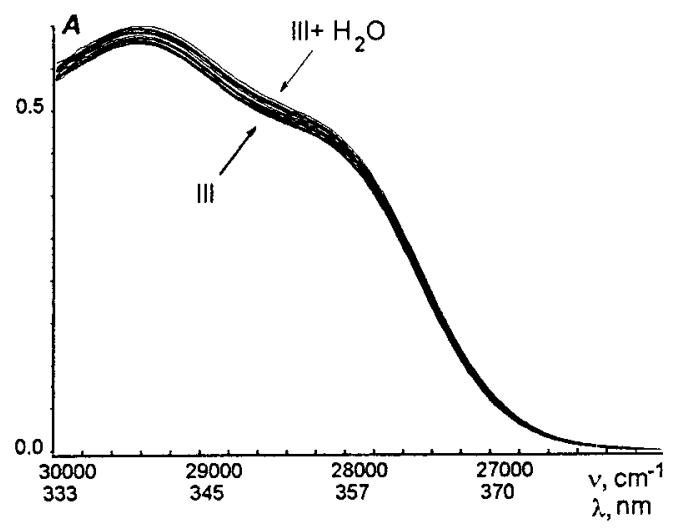

a

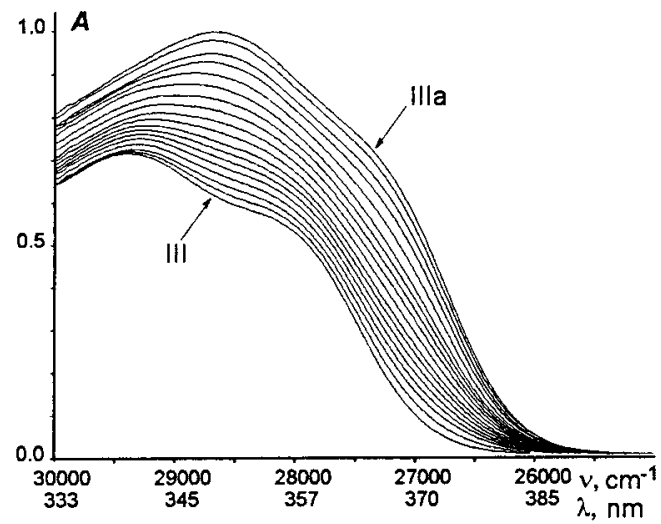

c

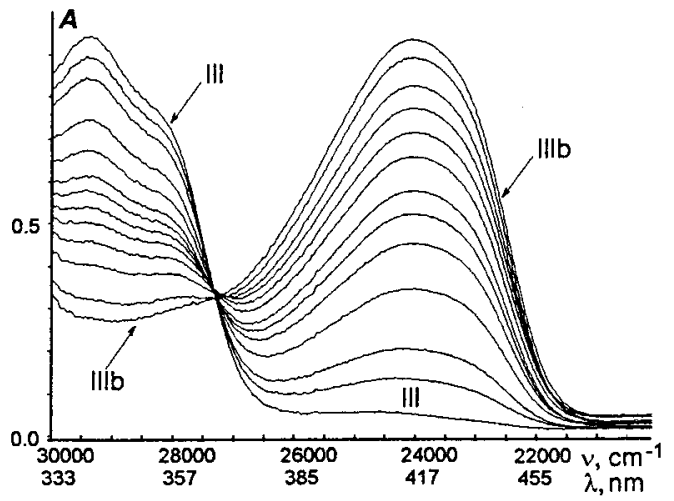

e

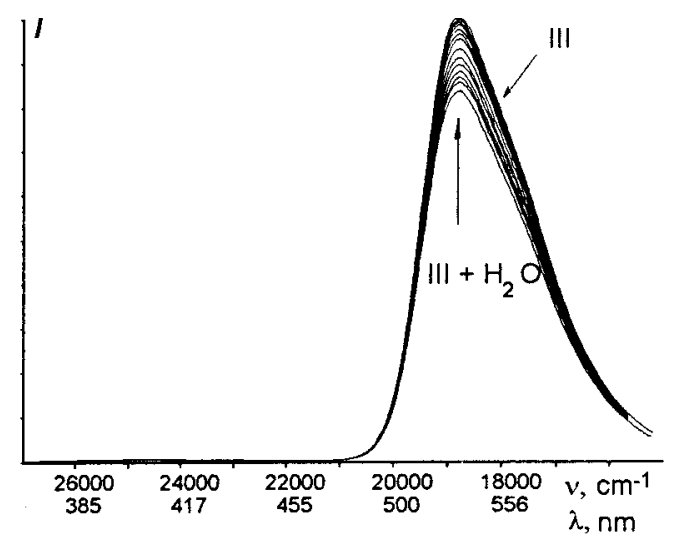

b

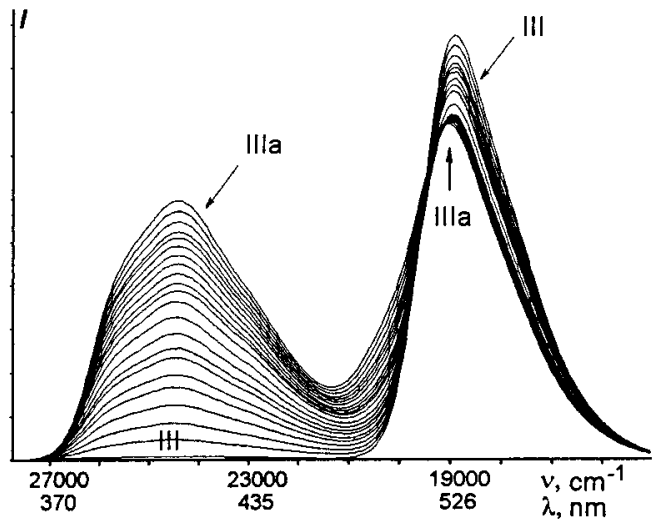

d

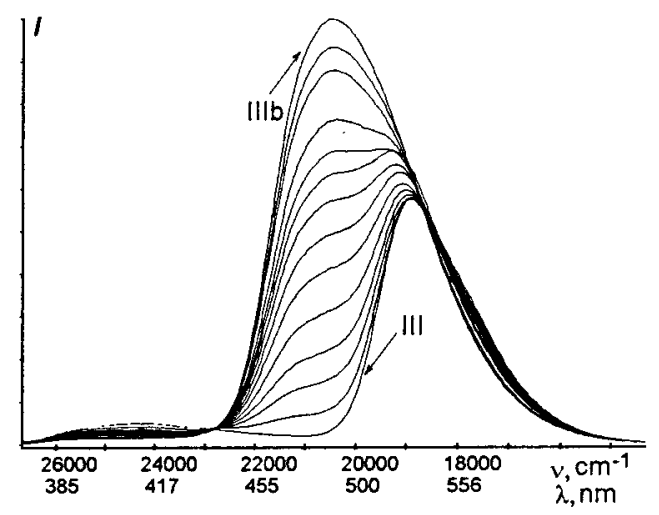

f

Figure 1. Changes in the absorption ( $a, c, e)$ and fluorescence spectra (b, d, f) of flavonol III on addition of water (a, b), barium perchlorate (c, $\mathrm{d}$ ), or magnesium perchlorate (e, f). The symbols representing complexes are the same as those used in Scheme 2.

of water on ion titration results enabled us to perform studies on the effect of water on ESIPT reaction in our compounds.

In Figure 1a,b the absorption and fluorescence spectra of flavonol III are presented. The spectra were recorded in acetonitrile with the addition of water in the concentration range from $2.0 \times 10^{-5}$ to $2.0 \mathrm{~mol} / \mathrm{L}$. We observe that opening of intramolecular hydrogen bonds and formation of intermolecular bonds with water molecules do not result in essential changes in absorption spectra: there occurs only a small bathochromic shift $\left(100-150 \mathrm{~cm}^{-1}\right)$ and some redistribution of intensities between vibronic components of the long-wavelength absorption band. In the fluorescence spectrum the decrease of intensity of the phototautomer form by only $10-15 \%$ is observed. It should be noticed that these changes occur at relatively high water content, and the constant of complex formation is low $\left(\log K_{\mathrm{S}}=0.17\right)$. The same observations take place in the experiments with flavonols I and II $\left(\log K_{\mathrm{S}}=-1.26\right.$ and -0.76 , respectively).

Complex Formation with Ba and Mg Ions. "External" Complexes. On the addition of alkaline-earth cations to flavones II-IV in acetonitrile the changes in their absorption and fluorescence spectra are more noticeable (Figure 1c-f), and 
TABLE 1: Spectroscopic Properties of Flavonols I-IV with $\mathrm{Mg}^{2+}$ and $\mathrm{Ba}^{2+}$ Cations

\begin{tabular}{|c|c|c|c|c|c|c|c|c|}
\hline \multirow[b]{2}{*}{ complex } & \multirow[b]{2}{*}{ ion } & \multicolumn{3}{|c|}{ absorption spectra } & \multicolumn{4}{|c|}{ fluorescence spectra } \\
\hline & & $\overline{\lambda, \mathrm{nm}}$ & $v, \mathrm{~cm}^{-1}$ & $\overline{\log \epsilon}$ & $\overline{\lambda, \mathrm{nm}}$ & $v, \mathrm{~cm}^{-1}$ & $\Delta v_{\mathrm{St}}, \mathrm{cm}^{-1}$ & $\varphi, \%$ \\
\hline \multirow[t]{2}{*}{ I } & & 399 & 25060 & 4.58 & 522 & 19150 & 5900 & 4.58 \\
\hline & & & & & $576^{* a}$ & 17370 & 7690 & 3.72 \\
\hline \multirow[t]{2}{*}{ Ic } & $\mathrm{Ba}^{2+}$ & 356 & 28080 & 4.64 & 522 & 19150 & 5900 & 4.58 \\
\hline & & & & & $576^{*}$ & 17370 & 7690 & 3.72 \\
\hline Ica & $\mathrm{Ba}^{2+}$ & 382 & 26180 & 4.57 & 535 & 18680 & 7500 & 46.8 \\
\hline Ib & $\mathrm{Mg}^{2+}$ & 444 & 22600 & 4.53 & 538 & 18600 & 4000 & 79.3 \\
\hline Ibc & $\mathrm{Mg}^{2+}$ & 419 & 29840 & 4.43 & 503 & 19880 & 3980 & 62.4 \\
\hline \multirow[t]{2}{*}{ II } & & 397 & 25180 & 4.53 & 527 & 18980 & 6200 & 6.48 \\
\hline & & & & & $578 *$ & 17300 & 7880 & 2.34 \\
\hline IIa & $\mathrm{Ba}^{2+}$ & 428 & 23340 & 4.73 & 548 & 18220 & 5120 & 56.3 \\
\hline IIb & $\mathrm{Mg}^{2+}$ & 448 & 22340 & 4.59 & 548 & 18240 & 4100 & 73.3 \\
\hline \multirow[t]{2}{*}{ III } & & 340 & 29400 & 4.11 & 406 & 24630 & 4810 & 0.09 \\
\hline & & & & & $530^{*}$ & 18860 & 10580 & 4.17 \\
\hline IIIa & $\mathrm{Ba}^{2+}$ & 350 & 28600 & 4.33 & 410 & 24420 & 3980 & 4.23 \\
\hline IIIb & $\mathrm{Mg}^{2+}$ & 420 & 23840 & 4.11 & 488 & 20480 & 3360 & 10.50 \\
\hline IV & & 296 & 33820 & 4.16 & & & & \\
\hline IVa & $\mathrm{Mg}^{2+}$ & 300 & 33280 & 4.56 & & & & \\
\hline IVa & $\mathrm{Ba}^{2+}$ & 304 & 32940 & 4.29 & & & & \\
\hline
\end{tabular}

$a *=$ spectroscopic properties of phototautomer.

together with the presence of isosbestic points, they provide evidence for complex formation. The basic spectroscopic properties of flavonols and their complexes are presented in Table 1. The studies of the dependence of absorption and emission spectra on ion concentration allow us to conclude that in all cases the flavonols II-IV exhibit 1:1 stoichiometry of binding.

By the character of spectroscopic changes, the complexes with metal cations can be classified into two groups. The complexes of the first type (Figure 1c) are formed on interaction of $\mathrm{Ba}^{2+}$ ions with flavonols II and III and also of $\mathrm{Ba}^{2+}$ and $\mathrm{Mg}^{2+}$ ions with 3-methoxyflavone IV. Long-wavelength bands in their absorption spectra are more intensive than in the case of absence of metal cations. In addition, the small bathochromic shifts are observed both in absorption and in fluorescence spectra. Stokes shifts $\left(\Delta \nu_{\mathrm{St}}\right)$ for complexes of this type are somewhat smaller than those for correspondent-free flavonols II-IV.

The similarity of spectroscopic properties of the complexes of the first type for flavonols II-III and 3-methoxyflavone IV elucidates the fact that the flavonol 3-OH group does not participate in complex formation. This conclusion is supported by the retention of ESIPT effect (the presence of a longwavelength phototautomer band) for the barium complex of flavonol III. In its fluorescence spectrum the phototautomer band is always present, up to the highest cation concentrations (Figure 1d). Thus it may be suggested that in the complexes of the first type (IIa-IVa) the proton of the flavonol hydroxylic group is not substituted by a metal cation. The metal cation forms a donor-acceptor linkage with the oxygen electron lone pair belonging to the $\mathrm{C}=\mathrm{O}$ group (Scheme 2 ).

The data presented in Table 1 demonstrate that the fluorescence spectroscopic properties of the complexes of the first type are similar to the properties of flavonols without cations. Therefore we can suggest that in this case the cation binding does not cause essential changes in the flavonol electronic structure. The stability of the barium complexes depends very little on the nature of the substituent in flavonol at positions 3 and $4^{\prime}$. Whether it is a hydroxy group or a methoxy group in position $3, \log K_{\mathrm{S}}$ is within the range $0.72-0.89$ (Table 2 ).

Thus, the 3-hydroxy group does not participate in the formation of complexes of the first type (Scheme 2). The metal cation forms a donor-acceptor bond with the "external" (not participating in the formation of intramolecular hydrogen bond)

\section{SCHEME 2}

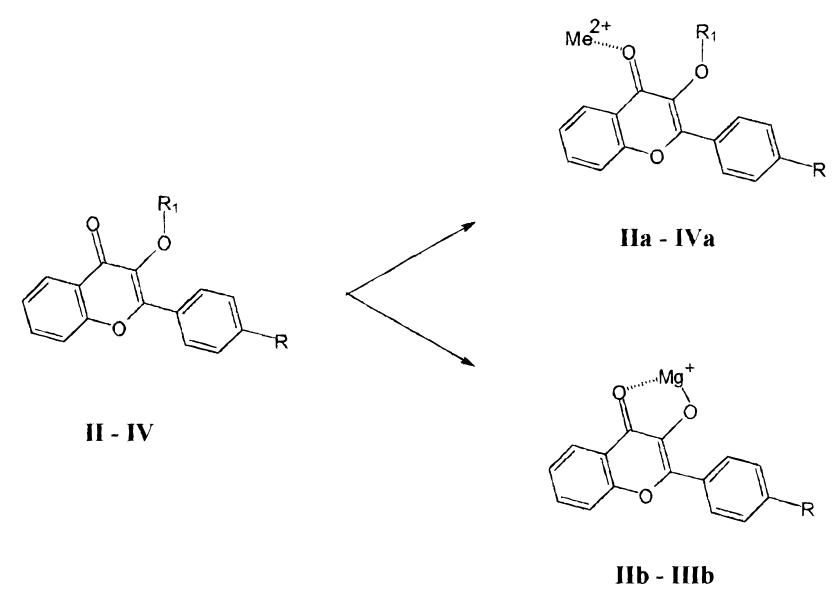

electron pair of the oxygen atom of the flavonol carbonyl group. Since this electron pair is located in the plane of the molecule, the contribution of the $\pi$-electronic system to the cation binding is minimal. This explains both a small influence of the $\pi$-electronic system on complex stability and the relatively weak polarizing effect of ion charge on this system, which results in only small electrochromic effects in absorption and fluorescence spectra.

Chelating Complexes of 3-Hydroxyflavones. In contrast to the data presented above, interaction of flavonols II and III with $\mathrm{Mg}^{2+}$ cations results in significant bathochromic shifts of both absorption and emission spectra (Figure 1e,f). The complexes Ib-IIIb, which are formed in this case, may be of different origin. They are observed only for 3-hydroxy-, but not for 3-methoxyflavones. Their stability constant is higher by 1 order of magnitude than that for the "external" complexes and depends on the substituent in the flavonol phenyl ring (Table 2). On this complexation the tautomer fluorescence band disappears completely. Thus, we can suggest that the flavonol hydroxylic group participates in the formation of $\mathbf{I b}$-IIIb complexes.

For these complexes the $\Delta v_{\mathrm{St}}$ values are approximately 1.5 times smaller than for the corresponding structures IIa-IVa. This fact points to the formation of a complex with more rigid molecular structure, whose conformation and interaction with the solvent do not change substantially in the excited state compared with the ground state. 
TABLE 2: Logarithm of Stability Constants $\left(K_{\mathrm{S}}\right)$ of Flavonols I-IV with $\mathrm{Ba}^{2+}$ and $\mathrm{Mg}^{2+}$ Ions

\begin{tabular}{|c|c|c|c|c|c|c|}
\hline type of complex & metal ion & I & II & III & IV & $\mathbf{V}$ \\
\hline $\mathbf{a}$ & $\begin{array}{l}\mathrm{Ba}^{2+} \\
\mathrm{Mg}^{2+}\end{array}$ & & $0.72 \pm 0.01$ & $0.76 \pm 0.10$ & $\begin{array}{l}0.75 \pm 0.04 \\
0.32 \pm 0.03\end{array}$ & \\
\hline b & $\mathrm{Mg}^{2+}$ & $2.25 \pm 0.07$ & $2.07 \pm 0.06$ & $1.71 \pm 0.02$ & & \\
\hline c & $\begin{array}{l}\mathrm{Ba}^{2+} \\
\mathrm{Mg}^{2+}\end{array}$ & $2.40 \pm 0.02$ & & & & $\begin{array}{l}3.57 \pm 0.02 \\
1.97 * \pm 0.09\end{array}$ \\
\hline $\begin{array}{l}\text { ca } \\
\text { bc }\end{array}$ & $\begin{array}{l}\mathrm{Ba}^{2+} \\
\mathrm{Mg}^{2+}\end{array}$ & $\begin{array}{l}0.89 \pm 0.04 \\
0.83 \pm 0.06\end{array}$ & & & & \\
\hline
\end{tabular}

TABLE 3: Distribution of Charge on the Fragments and Interfragment Charge Transfer in Flavonol Molecules

\begin{tabular}{|c|c|c|c|c|c|c|}
\hline & \multirow{3}{*}{$\begin{array}{c}\text { total charge } \\
\text { on the molecule }\end{array}$} & \multicolumn{4}{|c|}{ charges on the fragments } & \multirow{3}{*}{$\begin{array}{c}\text { transfer of charge, } \\
\Delta Q(\%)\end{array}$} \\
\hline & & \multicolumn{2}{|r|}{$\mathrm{S}_{0}$} & \multicolumn{2}{|r|}{$\mathrm{S}_{1}$} & \\
\hline & & phenyl & benzopyrone + ion & phenyl & benzopyrone + ion & \\
\hline I & 0 & +0.106 & -0.106 & +0.233 & -0.233 & 12.7 \\
\hline Ia & +1 & +0.207 & +0.793 & +0.595 & +0.406 & 38.7 \\
\hline III & 0 & +0.052 & -0.052 & +0.048 & -0.048 & 0 \\
\hline IIIa & +1 & +0.168 & +0.832 & +0.334 & +0.666 & 16.6 \\
\hline
\end{tabular}

These data allow us to suggest that the complexes of the second type are characterized by cyclic arrangement of chelates with magnesium ions (Scheme 2). Due to its larger size $\left(\mathrm{Baa}^{2+}\right.$ $=1.39 \AA$, compare with $\left.r_{\mathrm{Mg}^{2+}}=0.60 \AA\right)$, the barium cation cannot be located in the cavity between flavonol oxygen atoms of $\mathrm{C}=\mathrm{O}$ and $\mathrm{OH}$ groups (the radius of the cavity is approximated to be $0.80-0.90 \AA$ ) and therefore does not form chelates of this type.

The calculations of electronic structures of the complexes are in good agreement with this interpretation of experimental data. According to these calculations, the III b chelate formation with the $\mathrm{Mg}^{2+}$ ions results in substantial decrease of electronic density on the benzopyrone fragment. The distribution of charges on atoms of this bicyclic for the case of chelates in the $S_{0}$ and $S_{1}$ states is similar to the distribution for 2-phenylbenzopyrilic salts, especially for the salts of 3,4-dihydroxyflavilium, which are obtained on protonation of flavone III derivatives in media of high acidity. Thus, the transition $\mathrm{S}_{0} \rightarrow \mathrm{S}_{1}$ in chelate IIIb occurs between the states that are qualitatively different from that of flavonol. According to calculations, the difference in energy of boundary orbitals-HOMO and LUMO in the case of the IIIb complex - is less by about $1.5 \mathrm{eV}$ than for the corresponding orbitals of uncomplexed flavonol III. This explains the significant bathochromic shift of its long-wavelength absorption band on complex formation.

Introduction of a metal cation results not only in the increase of HOMO energy over that of LUMO but also in delocalization of the occupied orbital over the whole molecule (it is accounted that in the equation for the HOMO wave function there is a substantial contribution not only of the atomic orbitals of benzopyrone cycle atoms, as for flavonols, but also of atomic orbitals of the side phenyl ring). The wave function of the $S_{0}$ $\rightarrow \mathrm{S}_{1}$ transition may be described by the single excited configuration $\chi_{1 \rightarrow 1^{\prime}}$. As a result, this transition is associated with rather significant charge transfer from the side phenyl ring to the benzopyrone bicyclic (Table 3 ).

Analysis of localization of molecular orbitals for $4^{\prime}-\mathrm{N}, \mathrm{N}$ dimethylamino derivatives of 3-hydroxyflavone demonstrates that in the presence of an amino group in the phenyl ring, the HOMO is essentially localized on this ring and on the nitrogen atom. The nature of the LUMO does not change: the orbital is localized on the benzopyrone fragment. Thus the presence of a dimethylamino group in flavonols $\mathbf{I}$ and II results in formation of an excited state of charge-transfer character even in the absence of complexation. It can be assumed that the similar nature of the $S_{0} \rightarrow S_{1}$ transition is characteristic also for the complex of the first type, IIa, which is close to flavonol II by electronic structure.

Complex Formation by Crown-Flavonol. Crown-flavonol I and 4'-dimethylaminoflavonol II exhibit similar spectroscopic properties in acetonitrile solution. The absorption maximum of $\mathbf{I}$ is observed at $400 \mathrm{~nm}$, while the fluorescence maxima are at 522 and $576 \mathrm{~nm}$ (Table 1). As in the case of II, the spectroscopic parameters of $\mathbf{I}$ are determined to a significant extent by electron donor characteristics of the dialkylamino group.

The presence of small amounts of magnesium ions in acetonitrile solution of crown-flavonol I results in the appearance of a long-wavelength absorption band at $444 \mathrm{~nm}$ (Figure 2a).

On the grounds of our data obtained for compounds II-IV, which do not contain a crown group, it can be suggested that flavonol I with $\mathrm{Mg}^{2+}$ cations initially forms the chelation complex of the type Ib with the participation of 3-OH and 4-C= $\mathrm{O}$ oxygen atoms (Scheme 3 ).

On increase of the $\mathrm{Mg}^{2+}$ ion concentration, there begins the complex formation with the crown cyclic, which is associated with partial shift of the electron density of the nitrogen atom toward the metal cation. This effect results in a shortwavelength shift of the absorption band up to $25 \mathrm{~nm}$.

Similar behavior was typical for other azacrown ether dyes for which the complexation of the crown cyclic led to a decrease of electron donative ability of the azacrown group and also to a decrease of its influence on the main chromophoric fragment of studied molecules. ${ }^{25-27}$ However, complete disconjugation of the nitrogen atom and the $\pi$-electronic system, which is observed for protonation in acidic media, does not occur. The absence of such a disconjugation in our case is in line with the observation that the absorption spectra of magnium complexes Ibc are situated at longer wavelengths compared with the spectra of protonated flavonol and have a higher intensity.

The absorption spectra of Ib and Ibc demonstrate also that the binding of the second cation does not disrupt the complex with the first cation. Thus, the complex Ibc, which is formed at high $\mathrm{Mg}^{2+}$ concentrations, is probably of the type $2: 1$.

In the fluorescence spectrum of flavonol I the increase of $\mathrm{Mg}^{2+}$ concentration and the formation of chelating complex Ib result in the disappearance of fluorescence emission bands of both its normal form and of the phototautomer. Instead of them a new intensive band appears at intermediate wavelengths. The complex formation by the crown cyclic is revealed at the shortwavelength shift of this band to $503 \mathrm{~nm}$ and is followed by a slight decrease of fluorescence intensity (Figure 2b). Since the 


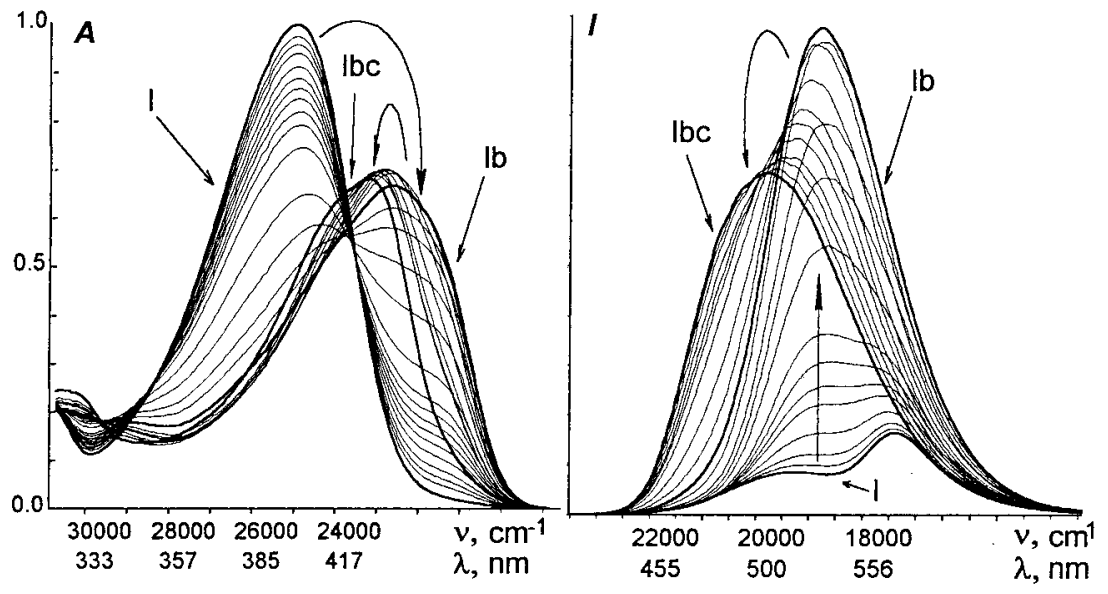

a

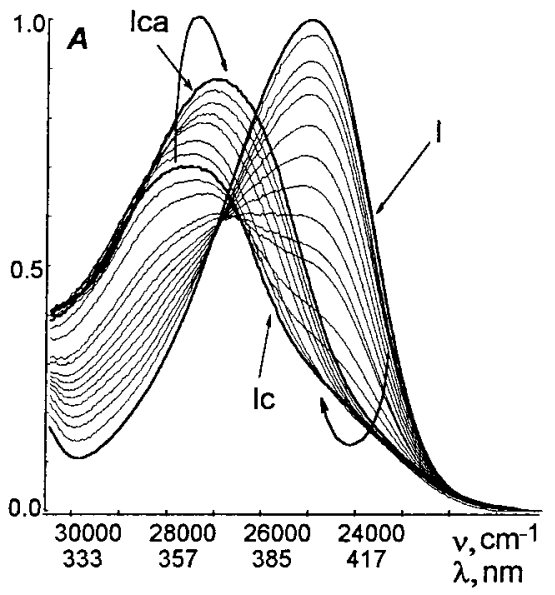

c b

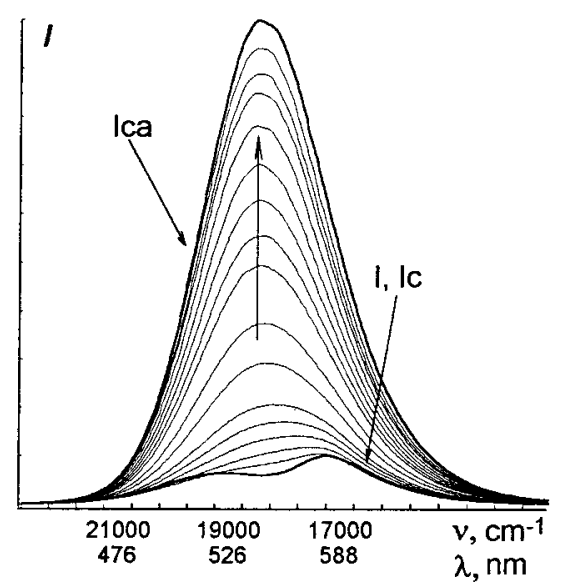

d

Figure 2. Changes in the absorption (a, c) and fluorescence spectra (b, d) of $4^{\prime}$-(15-azacrown-5)flavonol I on addition of magnesium perchlorate $(a, b)$ or barium perchlorate $(c, d)$. The symbols representing complexes are the same as those used in Scheme 3.

emission intensity in this case remains relatively high (by one order higher than that of a free flavonol I), it can be suggested that in the excited state the complex Ibc does not dissociate and exists with cation-flavonol stoichiometry of 2:1.

The complex formation of flavonol I with $\mathrm{Ba}^{2+}$ ions differs from the complex formation with $\mathrm{Mg}^{2+}$.

In the absorption spectrum of $\mathbf{I}$ on increase of concentration of barium ions we observe initially the appearance of a shortwavelength band at $356 \mathrm{~nm}$. This fact suggests that the first barium cation is bound to the crown group and the complex Ic is formed.

As in the case of complex Ibc formation with the $\mathrm{Mg}^{2+}$ ion, we could suppose that the significant hypsochromic shift of the long-wavelength absorption band of the Ic complex with barium ion is probably the result of the shift of electronic density from the nitrogen atom to the bound cation.

At higher $\mathrm{Ba}^{2+}$ ion concentrations the absorption maximum of complex Ic shifts to longer wavelength (to $385 \mathrm{~nm}$ ). In this case the second cation binds to the carbonyl oxygen atom and the complex Ica is formed, with 2:1 stoichiometry. Regarding the magnitude of the bathochromic shift of the absorption band $(26 \mathrm{~nm})$ and low stability constant of this complex $\left(\log K_{\mathrm{S}}=\right.$ 0.89), it may be suggested that the "external" complex with very small participation of the oxygen atom of the $3-\mathrm{OH}$ hydroxylic group was formed (Scheme 3).
The fluorescence spectrum of the Ic complex does not differ from the spectrum of free flavonol I. Therefore we may conclude that the complex Ic after electronic excitation dissociates, and fluorescence emission occurs from the same $S_{1}$ state of flavonol $\mathbf{I}$, which is characterized by substantial charge transfer from the side aromatic ring to the benzopyrone ring (Scheme 4). Similar dissociation of the complex in the excited state is observed also for other compounds that possess an azacrown group. ${ }^{25,26,28}$

More significant increase of $\mathrm{Ba}^{2+}$ ion concentration in solution results in the disappearance of the bands of flavonol I and the appearance of a new band of emission with high intensity and a maximum at $535 \mathrm{~nm}$. In our opinion, this emission band corresponds to an "external" complex, which does not exist in a ground state. This "external" complex is similar in structure to complex IIa with metal-ligand stoichiometry of $1: 1$. It should be noted that in this case the $\mathrm{Ba}^{2+}$ ion is bound with a carbonyl group, whereas the crown-cyclic is free.

The suggestion that flavonol I gives a complex similar to IIa is based on comparison of decomposition rates of "external" and of crown ether complexes. According to refs 26 and 29, lifetimes of $\mathrm{Li}^{+}$and $\mathrm{Ca}^{2+}$ complexes with 5-azacrown-15 merocyanine are 2 and $30 \mathrm{ps}$, respectively. The excited-state lifetime of the "external" complex IIIa is $1.8 \mathrm{~ns}$. So, it could be suggested that $\mathrm{Ba}^{2+}$ ion ejection from the crown-cyclic is approxi- 


\section{SCHEME 3}
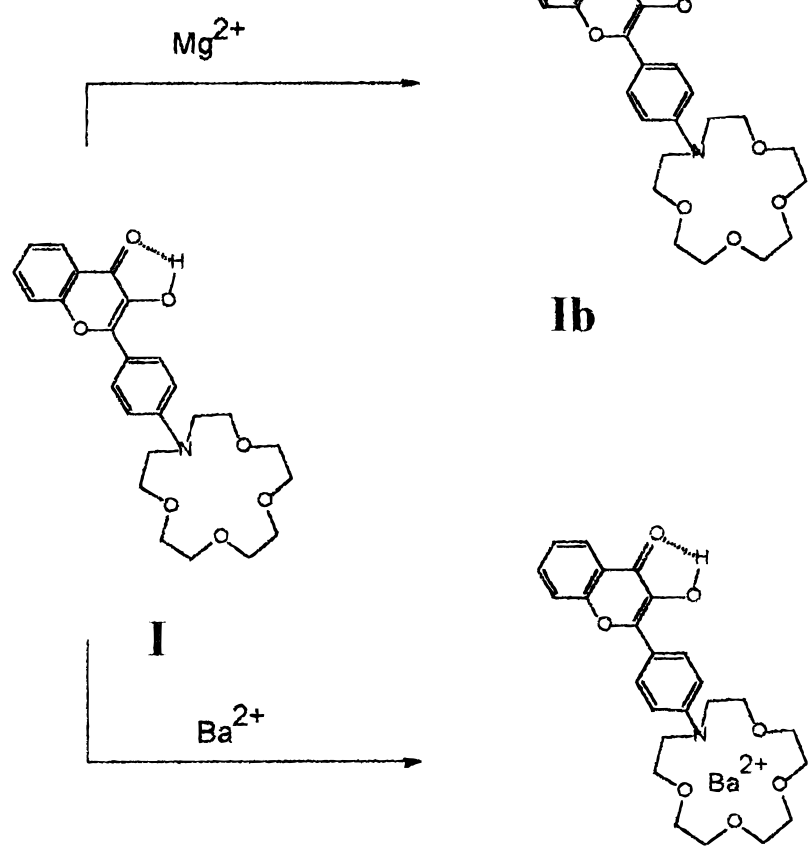

Ic

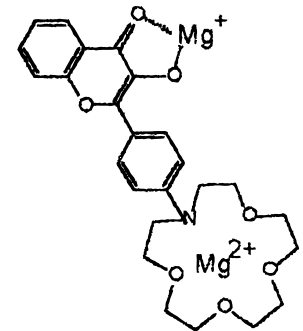

Ibc

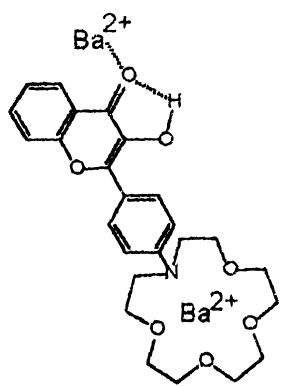

Ica

\section{SCHEME 4}

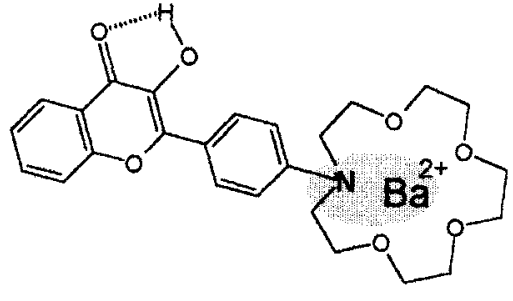

$\mathrm{S}_{0}$

matively $1.5-2$ orders faster than decomposition of the "external" complex of $\mathrm{Ba}^{2+}$ ion with the flavonol carbonyl group.

The difference in the ways of complexation of flavonol I with $\mathrm{Mg}^{2+}$ and $\mathrm{Ba}^{2+}$ ions (see Scheme 3 ) is determined not only by the difference between "external" and "internal" complex structures for these metals with 3-hydroxy and carbonyl groups but also by the stability of the crown-complexes formed.

It should be noted that the stability of a barium complex with model phenyl azacrown $\mathbf{V}$ is much higher than the stability of the corresponding magnesium complex (Table 2). This fact can be explained by better correspondence of the barium ion size than that of the magnesium ion to the diameter of a cavity of the crown cyclic. As a result, the interaction of a barium ion with crown cyclic oxygens is more effective.

The comparison of stability constants of barium complexes of crown-flavonol $\mathbf{I}$ and model azacrown $\mathbf{V}$ points out that the electronic state of nitrogen atoms of the crown cyclic influences the strength of complexes with metal cations. Thus, inclusion of an electron-accepting 3-hydroxybenzopyrone fragment into molecule $\mathbf{V}$ to give flavonol $\mathbf{I}$ results in a decrease of stability constant with metal cations by more than 1 order of magnitude (Table 2).

As was expected, the $\mathrm{Ba}^{2+}$ ion at first forms a more stable complex with the crown-cyclic and then forms a less stable

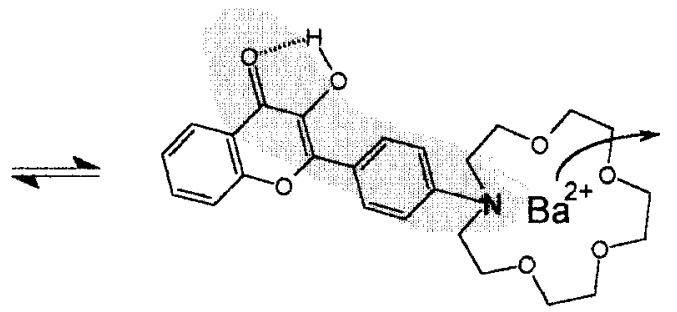

$\mathrm{S}_{1}$ "external" complex. In the case of the $\mathrm{Mg}^{2+}$ ion, the chelate complex is more stable and forms first.

Taking into account that "internal" and chelate complex stability nearly does not depend on flavonol type, it could be suggested that for ions of other metals the way of complexation with crown-flavonol would be determined by their ability to form chelating complexes and by the stability of the ion complexes with the crown-cyclic.

\section{Conclusions}

Flavonols and akaline-earth cations may form two types of complexes: low-stability "external" and high-stability chelating complexes. "Extenal" complexes are formed only by interaction with a metal cation of the sole electron pair of the oxygen atom belonging to the carbonyl group. In the formation of cyclic chelating complexes both the carbonyl group and the oxygen atom of of 3-hydroxy group participate.

Parent flavonols and 4'-dialkylamino flavonols behave differently on complex formation. In the former case the complex formation at the $3-\mathrm{OH}$ and $4-\mathrm{C}=\mathrm{O}$ sites results in considerable perturbation of electronic structure: the charge is transferred substantially from phenyl ring to the chromone part of the molecule. In the case of 4'-dialkylamino flavonols the com- 
plexes and free flavonols possess similar electronic composition both in the ground and in the excited states. In the excited state the charge transfer is observed for both free $4^{\prime}$-dialkylamino flavonol and its complexes with metal cations.

4'-(Aza-15-crown-5)-flavonol in acetonitrile forms two types of complexes with $\mathrm{Mg}^{2+}$ and $\mathrm{Ba}^{2+}$ cations with complex cation-flavonol stoichiometry of $1: 1$ or $1: 2$. The sequence of steps in complex formation is different. First the $\mathrm{Mg}^{2+}$ ion is bound in a chelating site formed by groups $3-\mathrm{OH}$ and $4-\mathrm{C}=\mathrm{O}$ and then is bound by the crown cyclic. In contrast, first the $\mathrm{Ba}^{2+}$ ion is bound with the crown cyclic and then forms the complex with the oxygen of carbonyl 4-C $=\mathrm{O}$. Magnesium complexes do not dissociate in the excited state substantially, while for barium ions the ejection of the cation from the crown complex is observed. The opposite sequence of interaction of the coordination centers of crown flavonol with $\mathrm{Mg}^{2+}$ and $\mathrm{Ba}^{2+}$ ions at complex formation leads to different changes in absorption and fluorescence spectra of this dye.

\section{References and Notes}

(1) Sengupta, P. K.; Kasha, M. Chem. Phys. Lett. 1979, 68, 382.

(2) McMorrow, D.; Kasha, M. J. Am. Chem. Soc, 1984, 88, 2235.

(3) Schwartz, B. J.; Peteanu, L. A.; Harris, C. B. J. Phys. Chem. 1992, 96, 3591.

(4) Swiney, T. C.; Kelley, D. F. J. Chem. Phys, 1993, 99, 211.

(5) Brucker, G. A.; Swinney, T. C.; Kelley, D. F. J. Phys. Chem. 1991, 95, 3190 .

(6) Sarkar, M.; Sengupta, P. Chem. Phys. Lett. 1991, 179, 68.

(7) Pivovarenko, V. G.; Tuganova, A. V.; Klimchenko, A. S.; Demchenko, A. P. Cell. Mol. Biol. Lett. 1997, 2, 355.

(8) Indicators; Bishop, E., Ed.; Pergamon Press: Oxford, 1972.
(9) Chou, P. T.; Martinez, M. L.; Clements, J. H. J. Phys. Chem. 1993, 97, 2618.

(10) Pivovarenko, V. G.; Roshal, A. D.; Demchenko, A. P. XVI IUPAC Symposium on Photochemistry; Helsinki, Abstracts, 1996, p 287.

(11) Pivovarenko, V. G.; Roshal, A. D.; Demchenko, A. P. Terenin Memorial International Symposium on Photochemistry and Photophysics of Molecules and Ions; St. Petersburg, Russia, Abstracts, 1996; Vol. B1, p 226.

(12) Hiraoka, M. Crown Compounds; Mir: Moscow, 1986.

(13) Gordon, A. J.; Ford, R. A. The Chemist's Companion; J. Wiley \& Sons: New York, 1972.

(14) Dean, F. M.; Podimuang, V. J. Chem. Soc. 1965, N.7, 3978.

(15) Smith, M. A.; Neumann, R. M.; Webb, R. A. J. Heterocycl. Chem. 1968, 5,425 .

(16) Löhr, H. G.; Vögtle, F. Acc. Chem. Res. 1985, 18, 65.

(17) The Methods of Preparation of High Purity Solvents; NIITEKHIM: Moscow, 1986

(18) Karyakin, Ju. V.; Angelov, I. I. Pure Chemical Compounds; Khimiya: Moscow, 1974.

(19) Ernst, Z. L.; Menashi, J. Trans. Faraday Soc. 1963, 59, Pt. 1, 230.

(20) Johnson, R. J.; Metzler, D. E. Methods Enzymol. 1970, 18a, 433

(21) Robinson, R. A.; Kiang, A. K. Trans. Faraday Soc. 1956, 52, 327.

(22) Bernstein, I. Ja.; Kaminskij, Yu. L. Spectrophotometric Analysis in Organic Chemistry; Khimija: Leningrad, 1986.

(23) Stewart, J. J. P. J. Comput. Chem. 1989, 10, 209

(24) Ormson, S. M.; Brown, R. G.; Vollmer, F.; Rettig W. J. Photochem. Photobiol. A: Chem. 1994, 81, 65.

(25) Druzhinin, S. J.; Rusalov, M. V.; Uzhinov, B. M.; Alfimov, M. V. Proc. Indian Acad. Sci. (Chem. Sci.) 1995, 107, 721.

(26) Martin, M.; Plaza, P.; Dai Hung, N.; Meyer, Y. H. Chem. Phys. Lett. 1993, 202, 425 .

(27) Takagi, M.; Ueno, K. Topics in Current Chemistry, Chemistry III: Host-Guest Complex; Plenium Press: New York, 1984; p 39.

(28) Valeur, B. Topics in Fluorescence Spectroscopy, vol. 4: Probe Design and Chemical Sensing; Plenium Press: New York, 1994, p 21.

(29) Martin, M.; Plaza, P.; Meyer, Y. H.; et al. J. Phys. Chem. 1996, 100,6879 